Ingeniería

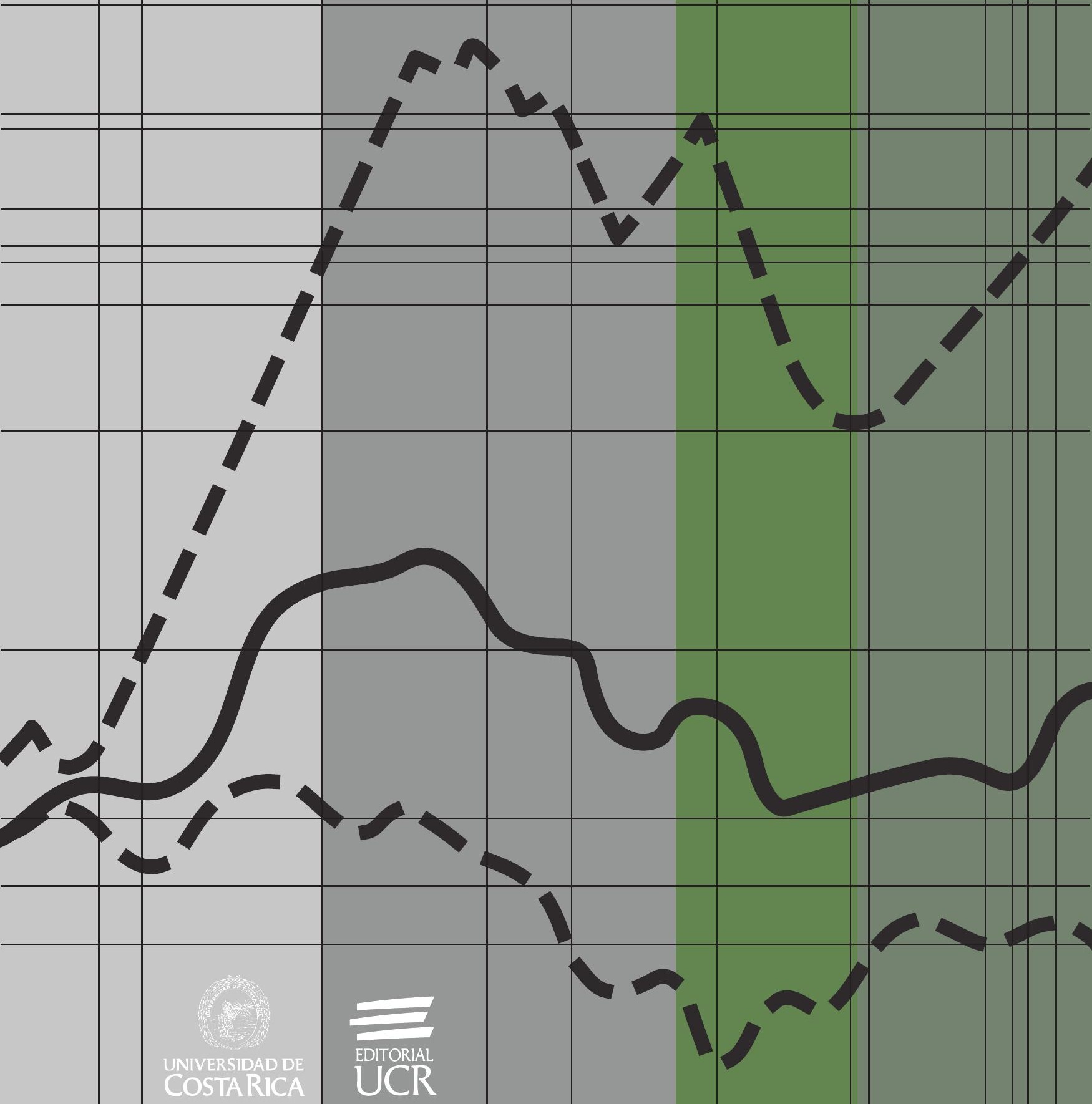




\title{
Desempeño mecánico de montajes de líneas de distribución de energía eléctrica
}

\author{
Mechanical Performance of Electric Power Distribution \\ Line Mounting \\ Raúl Fernández Vásquez. \\ Compañía Nacional de Fuerza y Luz, S.A.,Costa Rica \\ rfernandez@cnfl.go.cr
}

Recibido: 2 de abril 2018

Aceptado: 14 de mayo 2018

\begin{abstract}
Resumen
Inicialmente, mediante la revisión de normativa de otros países sobre los factores de seguridad para el diseño mecánico de líneas de distribución se concluye que lo más conveniente para las condiciones de la Compañía Nacional de Fuerza y Luz (CNFL) es la aplicación de los valores establecidos en el código de seguridad nacional de Estados Unidos de Norteamérica NESC. Seguidamente se realiza un análisis de esfuerzos en los montajes que se utilizan actualmente mediante modelado por elementos finitos obteniendo que para todos los casos, los montajes están subutilizados. También se realiza un análisis mediante equilibrio estático en los pernos que sujetan los montajes concluyendo que también son subutilizados.
\end{abstract}

\section{Palabras clave:}

Métodos de elemento finito, modelado y simulación de montajes, desempeño mecánico, media tensión, factores de diseño.

\begin{abstract}
Initially, by reviewing the regulations of other countries on the safety factors for the mechanical design of distribution lines, it is concluded that the most convenient conditions for Compañía Nacional de Fuerza y Luz (CNFL) is the application of the established values in the National Electrical Safety Code of the United States of America (NESC). Continually, an analysis of efforts is performed in the mounting that are currently used by modeling by finite elements, obtaining for all cases, the mounting are underutilized. An analysis is also performed by means of static equilibrium in the bolts that hold the mounting concluding that they are also underutilized.o matemático no lineal de Ratkowsky con un coeficiente de 0,971 y valores de $\alpha=11,04$ y $\beta=-1,49$ para la parametrización del modelo no lineal. Con los resultados obtenidos es posible predecir el valor de UFC presentes en el medio de cultivo para un valor determinado de F_0, lo cual puede ser de amplio interés en procesos de esterilización industrial, con el fin de ahorrar tiempo y costos de operación.
\end{abstract}


FERNÁNDEZ: Desempeño mecánico de montajes de líneas de distribución de energía...

Key Words:

Finite element method, electric assembly model and simulate, mechanical performance, medium voltage, design factors. 


\section{INTRODUCCIÓN}

En el inicio del desarrollo de los sistemas de distribución de energía eléctrica (incluso los de transmisión) los herrajes se fabricaban de madera, por lo que su formado era bastante limitado. A finales de los años setentas, cuando se dio el cambio al uso del acero en Costa Rica, la forma en esencia se mantuvo hasta los que se utilizan hoy en día. Este hecho sugiere que es posible que la forma en que están ordenados los elementos sea mejorable o existan diseños distintos que sean más convenientes.

En Costa Rica, desde hace más de 35 años se utilizan en la CNFL y otras empresas de distribución de energía eléctrica montajes conformados por pernos y herrajes fabricados a partir de perfiles de acero, específicamente perfil angular y rectangular. Por la gran experiencia acumulada, se sabe que los herrajes utilizados tienen suficiente capacidad para soportar las cargas mecánicas a las que usualmente son sometidos. Sin embargo, no se tiene conocimiento de la capacidad mecánica de cada uno de los montajes ni de cuál es el factor de seguridad en la construcción de estas estructuras por lo que no se tiene certeza de si se está abusando de sus características mecánicas o si por el contrario, se está subutilizando su capacidad.

Aunado a esto, en las últimas dos décadas ha sido necesario aumentar el calibre de los conductores para satisfacer la demanda de energía de una manera adecuada, incrementando con esto el peso de los conductores y por ende la tensión mecánica que soportan los montajes. Ante este escenario, es necesario determinar en qué condición de desempeño mecánico se encuentran actualmente los montajes utilizados en la CNFL. A partir de los resultados podrían realizarse mejoras importantes que vengan a optimizar el desempeño y eventualmente, reducir costos.

\section{MONTAJES DE LÍNEAS DE DISTRIBUCIÓN}

Los montajes de líneas de distribución de energía eléctrica son el conjunto de elementos que soportan los aisladores que mantienen la tensión eléctrica apartada de la tierra (eléctrica) y unen la línea de distribución al poste o estructura. En la figura 1(a) se muestra una línea de distribución convencional y en la 1(b) el conjunto de elementos que componen el montaje (exceptuando el poste), objeto de análisis de este artículo.

Existen tres tipos principales de montajes según el material principal, esto son: madera, concreto y acero. Desde el último cuarto del siglo XIX la madera fue el material más utilizado para dar soporte estructural a las líneas eléctricas, tanto el poste como el montaje, ya que además de brindar soporte, aportaban aislamiento eléctrico. Los montajes de madera son ampliamente utilizados en el mundo por su bajo costo inicial y menor impacto visual en zonas rurales, sin embargo, su desempeño y vida útil está directamente relacionado al mantenimiento que reciban. Los montajes de concreto son más robustos que los de madera y acero, tienen mayor capacidad mecánica, y por lo general son más altos y utilizados en diseños particulares donde se deban instalar conductores de mayor peso. Los montajes de acero por su parte se dividen en montajes de celosía, pre armados y de perfiles. Los montajes de celosía al igual que los de concreto, responden 
a diseños particulares donde las tensiones mecánicas son considerables, usualmente con largas distancias entre estructuras (300 m o más). Por su parte los montajes pre armados se diseñan por solicitud del cliente y responden a un diseño eléctrico que indica la separación, posición y altura de las faces (conductores). Finalmente, los montajes de perfiles están formados de partes denominadas herrajes y presentan la ventaja de que son versátiles (conformados por piezas separadas) y su costo es menor que el de los otros montajes de acero.

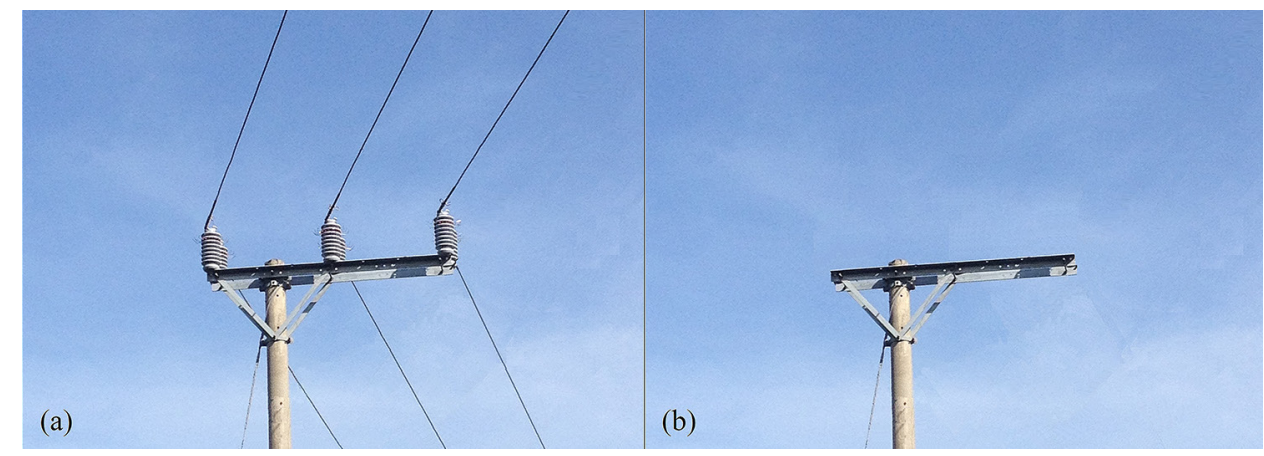

Figura 1 (a). Línea de distribución (b) Montaje de la línea.

Por las características particulares de la zona de servicio de la CNFL (zona urbana densamente poblada) es necesario que los montajes utilizados tengan suficiente versatilidad para hacer cambios y ajustes con una facilidad razonable ya que es común que se deban agregar nuevos tramos, cambiar la dirección o altura de los montajes por solicitudes de nuevos servicios o construcción de nuevas edificaciones por ejemplo. Esto hace que sean éstos los montajes utilizados por la CNFL.

\subsection{Composición de los montajes utilizados en la CNFL}

Los montajes trifásicos normalizados en la CNFL están formados por abrazaderas, cruceros, arriostres, soportes transversales, soportes para levantar aisladores y pernos. En la figura 2 se muestran cada uno de estos elementos (excepto el soporte para levantar aislador).

Los cruceros brindan soporte a los aisladores que sujetan los conductores, los arriostres brindan el soporte vertical y las abrazaderas son las que unen el montaje al poste. El soporte transversal es utilizado cuando la tensión mecánica es considerablemente distinta entre una dirección y la otra. El soporte para levantar aislador se utiliza para alejar la fase más cercana al poste en los montajes tipo bandera (adelante se detallan). Todos estos herrajes utilizan acero laminado tipo ASTM A36 (esfuerzo de fluencia $250 \mathrm{MPa}$ ) y algunos se fabrican a partir de angular y otros a partir de perfil rectangular. Actualmente en la CNFL se utilizan dos tipos de crucero, uno de $2 \mathrm{~m}$ de longitud y otro de 2,4 m. Así mismo, se utilizan dos tipos de arriostre dependiendo del soporte que se requiera. 
Finalmente, los montajes son armados con pernos ASTM A307 grado A y utilizan dos clases de aislador, los tipo remate y los tipo poste vertical. En la figura 3 se muestran los dos tipos de aisladores.

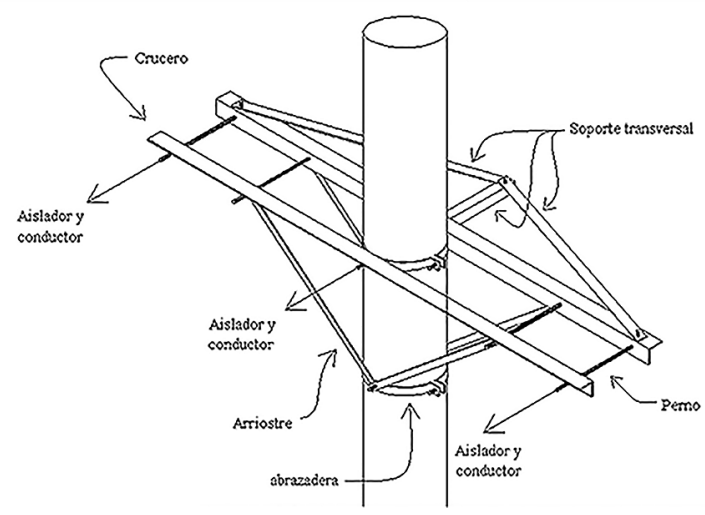

Figura 2. Herrajes utilizados en los montajes.

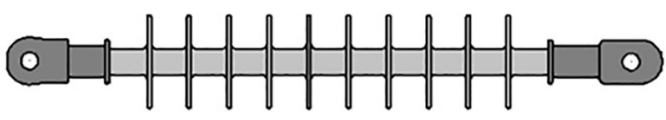

Tipo remate

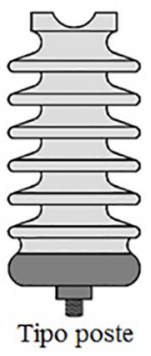

Figura 3. Aisladores utilizados en la CNFL.

Para el desarrollo del estudio se consideró como punto base el poste por lo que no se analiza esta estructura.

\subsection{Montajes normalizados en la CNFL}

Los montajes de las líneas trifásicas de distribución se clasifican de acuerdo al ángulo de desvío de la línea y al calibre del conductor que soporten. En la figura 4 se muestra la definición de ángulo de desvío.

En cuanto al calibre, en la CNFL se utilizan únicamente 4 tipos de conductores y en función de éstos, se utiliza uno u otro crucero (elemento que brinda la separación entre fases). Para conductores $2 \mathrm{AWG}$ o $3 / 0$ AWG se utilizan cruceros de $2 \mathrm{~m}$ (se denominan montajes livianos) y para conductores $266 \mathrm{AAC}$ o $477 \mathrm{AAC}$ se utilizan cruceros de 2,4 m (montajes pesados).

Los montajes trifásicos normalizados en la CNFL son: paso trifásico de $0^{\circ}$ a $10^{\circ}$, paso trifásico de $10^{\circ}$ a $30^{\circ}$, paso trifásico en bandera de $0^{\circ}$ a $10^{\circ}$, paso trifásico en bandera de $10^{\circ}$ a $30^{\circ}$, abertura trifásica y remate trifásico. 


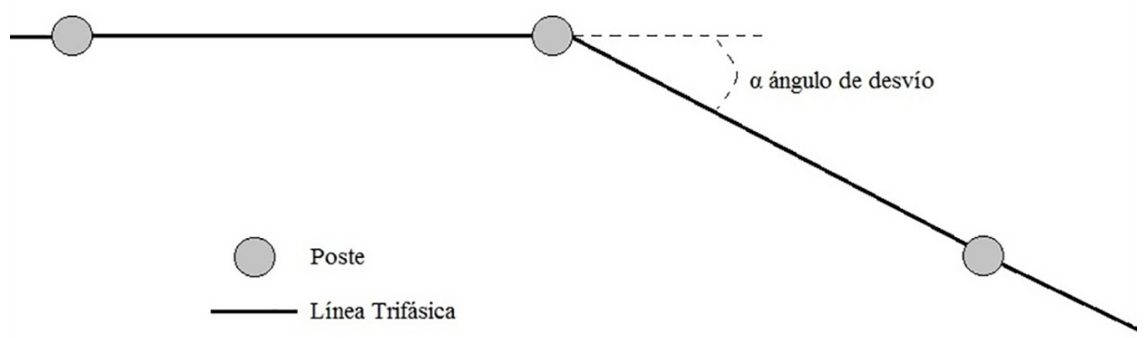

Figura 4. Vista en planta del ángulo de desvío.

En los primeros 4 montajes, el ángulo es el máximo de desvío de la línea, el montaje abertura trifásica se utiliza cuando el ángulo de desvío es mayor a $30^{\circ}$ y menor que $60^{\circ}$ o cuando se desea abrir la línea en el poste para efectos principalmente constructivos y por último, el montaje de remate se utiliza para finales de línea o ángulos de desvío superiores a $60^{\circ}$. En los primeros cuatro casos, la tensión es transmitida a los cruceros por los aisladores tipo poste vertical, mientras que en los últimos dos, la tensión de los conductores es transmitida a los pernos mediante los aisladores tipo remate. En la figura 5 se muestra un esquema de los montajes normalizados en la CNFL.

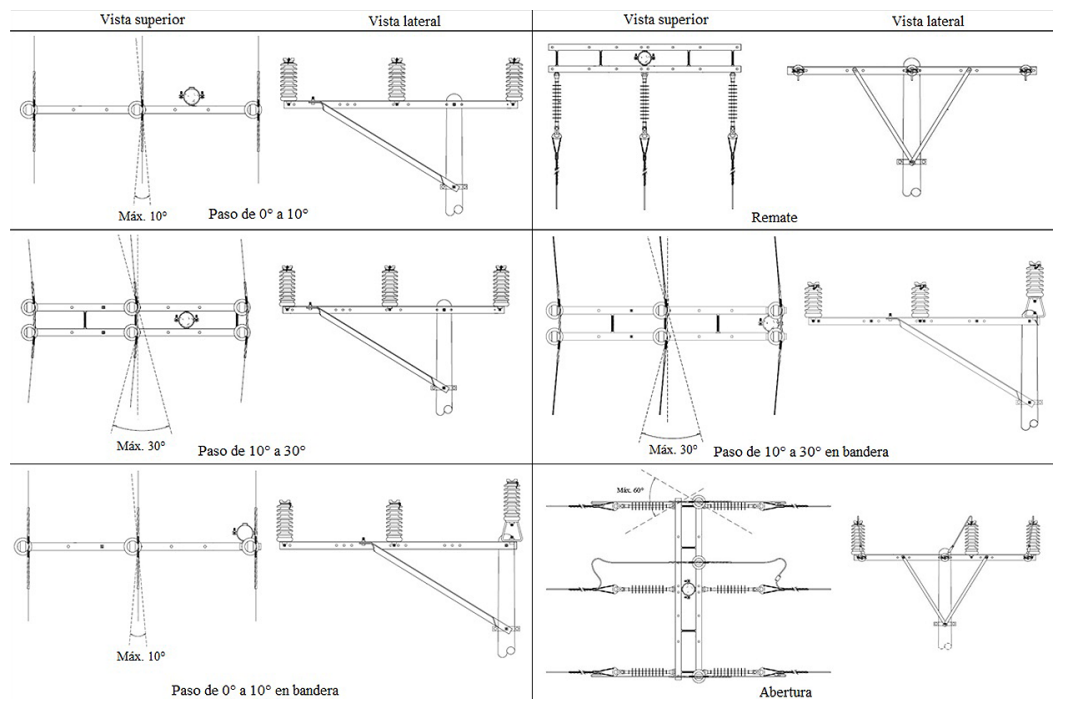

Figura 5. Montajes normalizados.

Cada uno de los montajes de la figura 5 se divide en dos de acuerdo al tipo de conductor (liviano o pesado) para un total de 12 montajes analizados.

\subsection{Fuerzas}

Bajo condiciones normales de operación, los montajes se ven sometidos a dos fuerzas: la tensión y peso de los conductores y el peso de los aisladores. También pueden verse sometidos a otras fuerzas como el peso de una persona de pie sobre los mismos (es común 
que en el proceso de instalación el personal técnico se apoye en los montajes para realizar la instalación de aisladores y conductores). La tensión ejercida por los conductores al montaje no es constante, la misma es dependiente de la tensión de instalación y varía en función de la temperatura (por la expansión térmica de los conductores) y de la velocidad del viento (con el aumento en la velocidad del viento aumenta la tensión ejercida por los conductores al montaje). La tensión de instalación de los conductores depende a su vez de la distancia entre postes y del tipo de montaje. De acuerdo a lo indicado en el apartado 2.2 , los conductores se pueden clasificar en dos tipos, siendo el conductor más robusto del tipo pesado el 477 AWG y el calibre 3/0 AWG el más robusto del tipo liviano por lo que son estos los utilizados para todos los cálculos de esfuerzos. Según los criterios para el diseño mecánico de líneas de distribución de la CNFL indicados por Fernández (2014), la tensión más elevada a la que se verá sometido un montaje es la que se presenta en condiciones de máximo viento $(60 \mathrm{~km} / \mathrm{h})$ y mínima temperatura $\left(10^{\circ} \mathrm{C}\right)$ cuando la separación entre postes es de 60 m y la tensión de instalación de $12 \%$ la tensión de rotura del conductor. Por su parte, el peso máximo (esfuerzo vertical) de los conductores en los montajes, es también el que se presenta cuando la separación entre postes es de $60 \mathrm{~m}$. En la tabla 1 se muestran las tenciones de diseño para cada una de las clases de montaje.

Tabla 1. Condiciones de diseño para cada clase de montaje. Fernández (2014).

\begin{tabular}{cccccc}
\hline \multirow{2}{*}{ Tipo } & $\begin{array}{c}\text { Conductor de } \\
\text { diseño }\end{array}$ & $\begin{array}{c}\text { Tnstalación } \\
\left(\text { sin viento y } 25^{\circ} \mathrm{C}\right)\end{array}$ & $\begin{array}{c}\text { Máxima } \\
\left(60 \mathrm{~km} / \mathrm{h} \mathrm{y} 10^{\circ} \mathrm{C}\right)\end{array}$ & $\begin{array}{c}\text { Montaje de } \\
\text { remate }\end{array}$ & $\begin{array}{c}\text { Montaje de } \\
\text { paso }\end{array}$ \\
\hline Pesado & $477 \mathrm{AAC}$ & $4550 \mathrm{~N}$ & $7140 \mathrm{~N}$ & $200 \mathrm{~N}$ & $400 \mathrm{~N}$ \\
Liviano & $3 / 0 \mathrm{AAC}$ & $1650 \mathrm{~N}$ & $2750 \mathrm{~N}$ & $70 \mathrm{~N}$ & $140 \mathrm{~N}$ \\
\hline
\end{tabular}

\subsection{Factores para el diseño}

Para determinar el o los factores de seguridad más recomendables para este tipo de estructuras, se consultó normativa y estándares constructivos y de diseño de distintos países. Al respecto, conviene definir los distintos factores utilizados.

El factor de seguridad se define como establece la ecuación 2.1.

$$
F . S .=\frac{S_{\max }}{S_{y}}
$$

Donde $\mathrm{S}_{\max }$ es el esfuerzo máximo que pueda presentarse y Sy el esfuerzo de fluencia del elemento en cuestión. Algunos estándares definen el factor de seguridad como la relación entre el esfuerzo máximo y el de rotura del elemento. Otro factor utilizado para el diseño es el de sobrecarga, el cual se define como sigue: 


$$
\text { Fact }_{\text {sobrec }}=\frac{F_{\text {diseño }}}{F_{\max }}
$$

Donde $\mathrm{F}_{\max }$ es la fuerza máxima que puede ser aplicada al elemento (condiciones críticas), Factsobrec es el factor de sobrecarga, que es un multiplicador que debe ser aplicado a la fuerza máxima para obtener la fuerza de diseño $\mathrm{F}_{\text {diseño }}$, que debe ser la utilizada para el análisis.

También conviene definir el factor de esfuerzo Fact esf $_{\text {, }}$ que es la relación entre el esfuerzo que debe ser utilizado para el diseño $S_{\text {diseño }}$ y el esfuerzo de fluencia del material $\mathrm{S}_{\mathrm{y}}$.

$$
\text { Fact }_{e s f}=\frac{S_{\text {diseño }}}{S_{y}}
$$

Tanto el factor de seguridad como el sobrecarga son mayores a 1, el factor de esfuerzo es igual o menor a la unidad. A continuación se detallan los datos más relevantes de cada una de las fuentes consultadas.

- El National Electric Safety Code NESC (2002) de Estado Unidos, define dos factores de importancia: el factor de sobrecarga y el factor de esfuerzo. El de esfuerzo para elementos de acero y pernos es de 1,0 y el de carga es 1,5 para esfuerzos verticales y 1,1 para esfuerzos horizontales.

- Las Normas de diseño de redes de distribución de energía eléctrica, de la Empresa de Energía de Boyacá S.A. EBSA (2010) de Colombia define el factor de seguridad como la máxima tensión sobre la resistencia del elemento (esfuerzo rotura). Esta norma establece que para herrajes y pernos el factor de seguridad debe ser de 3,0.

- En la Norma: Bases para el diseño de líneas y redes secundarias para la electrificación rural, de la Dirección General de Electricidad DGE (2003) del Ministerio de Energía y Minas de Perú se establece que el factor de seguridad para herrajes y tornillería debe ser de 2. En este caso, al igual que en el anterior, se indica que el factor de seguridad es sobre el esfuerzo último de los materiales.

- El Reglamento de Líneas Aéreas de Alta Tensión de España (1968) establece para los herrajes sometidos a tensión mecánica un factor de seguridad de 2,5 sobre la tensión de rotura del elemento. Éste documento se refiere a herrajes utilizados para líneas de transmisión.

- La Empresa Provincial de Energía de Córdoba (1998) de Argentina, establece un factor de seguridad de 2,0 referido a la tensión de rotura de los herrajes utilizados en líneas de distribución de energía eléctrica. 
- La empresa ENERSIS, donde participan las empresas CERJ y COELSE de Brasil, EDENOR y EDESUR de Argentina, CHILECTRA de Chile y CODENSA de Colombia en el documento Convergencia de Criterios de Diseño de Líneas Aéreas de Alta Tensión (2002) establece un factor se seguridad de 3,0 para los herrajes. El documento establece que este factor es a la rotura. Éste documento se refiere a herrajes utilizados para líneas de transmisión.

Tomando en cuenta los procesos de compras de la CNFL, donde se establecen y aseguran requisitos mínimos (el esfuerzo de fluencia es uno de ellos), se adopta la metodología del NESC para la evaluación del desempeño mecánico de los montajes que se utilizan en la actualidad.

Para el acero ASTM A36, como se indicó en el apartado 2.1, el esfuerzo de fluencia es de $250 \mathrm{MPa}$ por lo que es éste el parámetro considerado como máximo aceptable para el diseño. En el caso de los pernos, es el esfuerzo de fluencia establecido en la norma ASTM A307, que corresponde a $250 \mathrm{MPa}$ cuando la fuerza esté alineada con el perno (tensión pura). En caso de que el perno sea sometido a flexión, se utilizará $320 \mathrm{MPa}$ como límite de fluencia según lo indicado por Soltis (1996), y 100 MPa para esfuerzo cortante de acuerdo a Shigley (2002).

A partir las tensiones mostradas en la tabal 1, los montajes mostrados en la figura 5 y los factores de sobrecarga establecidos, se determinaron las fuerzas para el modelado de esfuerzos de los montajes, las cuales se muestran en la tabla 2.

Tabla 2. Fuerzas aplicadas a cada montaje con factor de sobrecarga.

\begin{tabular}{llll}
\hline Montaje - tipo & Fuerza aplicada en & \multicolumn{2}{c}{ Fuerza } \\
\hline Paso $0^{\circ}$ a $10^{\circ}$ - liviano & Cúspide de los 3 aisladores & $375 \mathrm{~N}$ & $528 \mathrm{~N}$ \\
Paso $0^{\circ}$ a $10^{\circ}$ - pesado & Cúspide de los 3 aisladores & $765 \mathrm{~N}$ & $1370 \mathrm{~N}$ \\
Paso $10^{\circ}$ a $30^{\circ}$ - liviano & Cúspide de los 6 aisladores & $270 \mathrm{~N}$ & $790 \mathrm{~N}$ \\
Paso $10^{\circ}$ a $30^{\circ}$ - pesado & Cúspide de los 6 aisladores & $465 \mathrm{~N}$ & $2050 \mathrm{~N}$ \\
Paso $0^{\circ}$ a $10^{\circ}$ bandera - liviano & Cúspide de los 3 aisladores & $375 \mathrm{~N}$ & $528 \mathrm{~N}$ \\
Paso $0^{\circ}$ a $10^{\circ}$ bandera - pesado & Cúspide de los 3 aisladores & $765 \mathrm{~N}$ & $1370 \mathrm{~N}$ \\
Paso $10^{\circ}$ a $30^{\circ}$ bandera - liviano & Cúspide de los 6 aisladores & $270 \mathrm{~N}$ & $790 \mathrm{~N}$ \\
Paso $10^{\circ}$ a $30^{\circ}$ bandera - pesado & Cúspide de los 6 aisladores & $465 \mathrm{~N}$ & $2050 \mathrm{~N}$ \\
Abertura - liviano & 6 caras de los 4 pernos & $105 \mathrm{~N}$ & $3025 \mathrm{~N}$ \\
Abertura - pesado & 6 caras de los 4 pernos & $300 \mathrm{~N}$ & $7854 \mathrm{~N}$ \\
Remate - liviano & Cara de los 3 pernos & $105 \mathrm{~N}$ & $3025 \mathrm{~N}$ \\
Remate - pesado & Cara de los 3 pernos & $300 \mathrm{~N}$ & $7854 \mathrm{~N}$ \\
\hline
\end{tabular}

${ }^{a}$ Más $165 \mathrm{~N}$ correspondientes al peso de los aisladores tipo poste vertical por el respectivo factor de sobrecarga, aplicada en la cúspide de los mismos. 


\section{DESEMPEÑO DE LOS MONTAJES UTILIZADOS EN LA CNFL}

Como se puede apreciar en las figuras 2 y 5 , los montajes son sujetados al poste mediante abrazaderas y pernos. Con el fin de simplificar el modelado, se analiza el comportamiento de las abrazaderas y los pernos por separado. Inicialmente se analizan los herrajes de cada montaje en conjunto mediante el modelado de esfuerzos por elementos finitos. Posteriormente se determina el esfuerzo transmitido a la sujeción (perno y abrazadera) mediante análisis de equilibrio estático y finalmente se analiza la abrazadera por medio del modelado por elementos finitos.

\subsection{Modelado de los montajes}

El modelado de los montajes se realiza con COMSOL Multiphysics. Los detalles relevantes del modelado se muestran a continuación.

Los aisladores tipo poste se modelaron como cilindros de un diámetro igual al de la base con el fin de simular la transferencia de la tensión de los conductores de manera semejante a la real. Las tensiones fueron aplicadas en la cara de los pernos o parte superior de los aisladores según correspondiera (ver tabla 2).

En la figura 6a se muestra el diagrama del montaje paso trifásico de $0^{\circ}$ a $10^{\circ}$ y la aplicación de la tensión T que ejercen los conductores en los aisladores. En la figura $6 \mathrm{~b}$ se muestra el diagrama de cuerpo libre para este mismo montaje. Para todos los casos donde la tensión es ejercida en los aisladores tipo poste vertical, se sumó esa componente con el peso de esos elementos. Las reacciones R1 y R2 son las ejercidas en los pernos de soporte y sujeción a las abrazaderas (referencia para la simulación). Las mismas son determinadas posteriormente por análisis de equilibrio estático.

a)

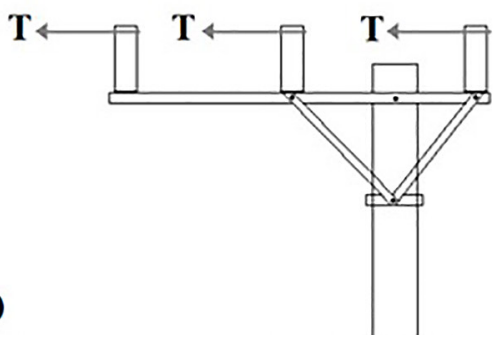

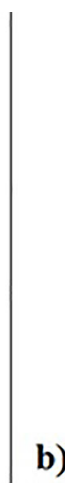

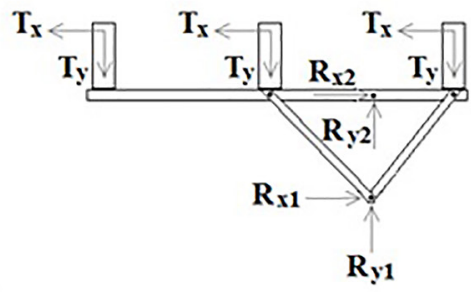

Figura 6. Diagrama de cuerpo libre del montaje paso de $0^{\circ}$ a $10^{\circ}$.

Para este montaje, en la figura 7 se muestra la aplicación en COMSOL Multiphysics de la tensión en el cilindro que modela el aislador. La sujeción o referencia fija se aplica en la cara interna de los pernos que sujetan el montaje a la abrazadera, esto por ser éste el punto donde se transfiere la fuerza del montaje a la abrazadera. En el recuadro de la figura 7 se muestra esta sujeción. En los casos en que esta sujeción produjo concentración 
de esfuerzos elevada (producto de la misma sujeción), se utilizó el perno completo como referencia fija para mejorar la escala y visualización de los resultados.

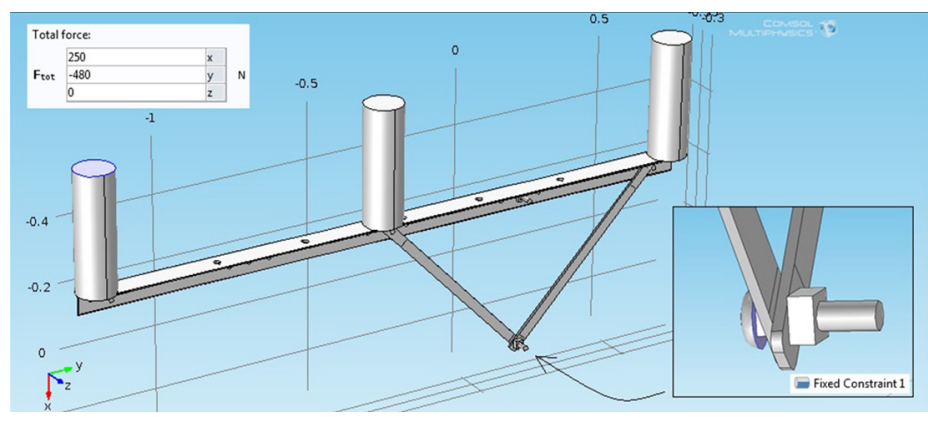

Figura 7. Detalles de sujeción y aplicación de las fuerzas.

De acuerdo a lo expuesto en la sección 2.1, se utilizó acero ASTM A36 para todos los perfiles y acero ASTM A307 para los pernos. Las fuerzas utilizadas para el modelado son las mostradas en la tabla 2, que consideran el factor de sobrecarga. Con respecto al mallado, para cada montaje se realizaron simulaciones para distintas combinaciones del tamaño mínimo y máximo de elemento así como de tasa de crecimiento de malla, con el fin de utilizar la más conveniente y evitar resultados viciados producto de una inadecuada elección de la malla.

A continuación se detallan los resultados obtenidos para el montaje de paso en bandera de $10^{\circ}$ a $30^{\circ}$ tipo pesado y para el montaje de remate pesado. Posteriormente se resumen los resultados de todos los montajes analizados.

\section{Paso de $10^{\circ}$ a $30^{\circ}$ en bandera pesado}

En la figura 8 se muestran los resultados de simulación de las condiciones críticas con un factor de escala de 10 .

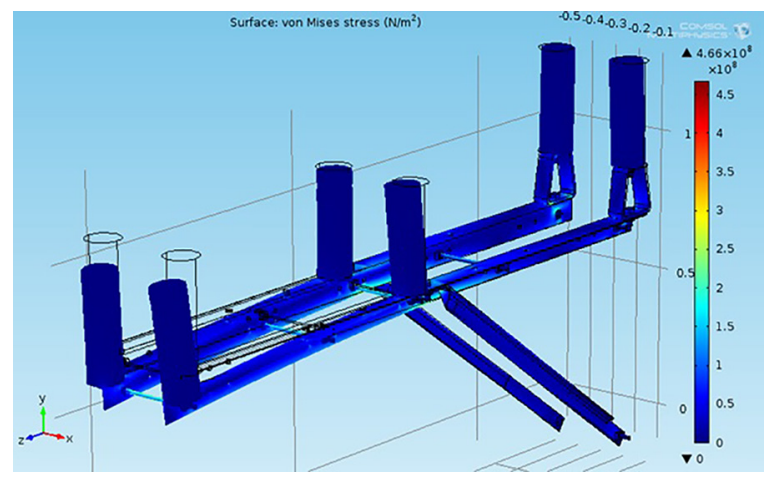

Figura 8. Condiciones críticas - Paso $10^{\circ}$ a $30^{\circ}$ bandera pesado. 
A pesar de que el modelado indica la existencia de un esfuerzo de $466 \mathrm{MPa}$, éstos corresponden a esfuerzos en el crucero contiguo al perno de referencia fija por lo que no es un resultado que represente la realidad. Para este caso, los esfuerzos de tensión más elevados se encuentran en los pernos de armado doble, específicamente, en la parte inferior del perno el más lejano al poste (174 MPa) y el central (154 MPa), mostrados en la figura 9-a y b respectivamente. En cuanto a los herrajes, es en la parte superior del crucero, en las cercanías con los arriostres que se presenta el mayor esfuerzo de tensión (161 MPa). En la figura 9-c se muestra el primer esfuerzo principal de estas zonas.

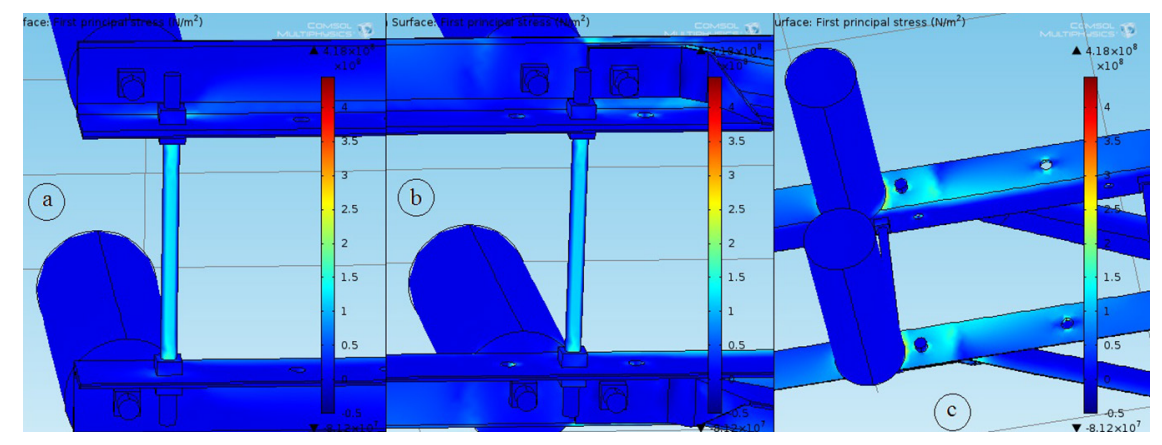

Figura 9. Esfuerzos de tensión - Paso $10^{\circ}$ a $30^{\circ}$ bandera pesado.

Adicionalmente, en el arriostre, específicamente en las cercanías a la unión con el crucero, se presenta un esfuerzo de $159 \mathrm{MPa}$. Esa zona también se observa en la figura 11-c. Para este montaje el mayor desplazamiento que se presenta en condiciones de sobrecarga es de 21,7 mm en los aisladores más lejanos al poste.

\section{Remate pesado}

Este es el montaje con mayor carga mecánica. La tensión de las líneas de distribución en este montaje está aplicada en una única dirección y bajo condiciones de sobrecarga es de $7854 \mathrm{~N}$ en la componente horizontal y $300 \mathrm{~N}$ en la componente vertical y es aplicado en los pernos extremos y el central.

Este montaje es el que utiliza el soporte transversal para contrarrestar la tensión mecánica ejercida por los conductores. Este elemento presentó una limitante en el modelado, por la condición particular de simulación como unión (y no como ensamble) las pletinas del soporte transversal se ven sometidas a pandeo obteniendo resultados que se alejan de la realidad. Esta condición se puede ver en la figura 10-a. En condiciones reales las platinas pueden girar sobre el eje de los pernos que los unen al montaje, evitando el pandeo y sometiéndolas únicamente a tensión. Para evitar este resultado, se modeló el montaje con el soporte transversal rígido, siendo esta una condición más crítica que la real. En la figura 10-b se puede ver el resultado de esta simulación. Tanto la figura 10-a como la 10-b tienen un factor de escala de las deformaciones de 20.

Como se puede ver, el máximo esfuerzo que se presenta en este montaje es de 242 $\mathrm{MPa}$, sin embargo, corresponde a un esfuerzo de compresión. El máximo esfuerzo de 
tensión que se presenta en este montaje es de $229 \mathrm{MPa}$ y se da en los pernos de armado doble extremos. En el caso del perno central, el esfuerzo es de $98 \mathrm{MPa}$. En la figura 11 se muestra el detalle del primer esfuerzo principal en los pernos mencionados.

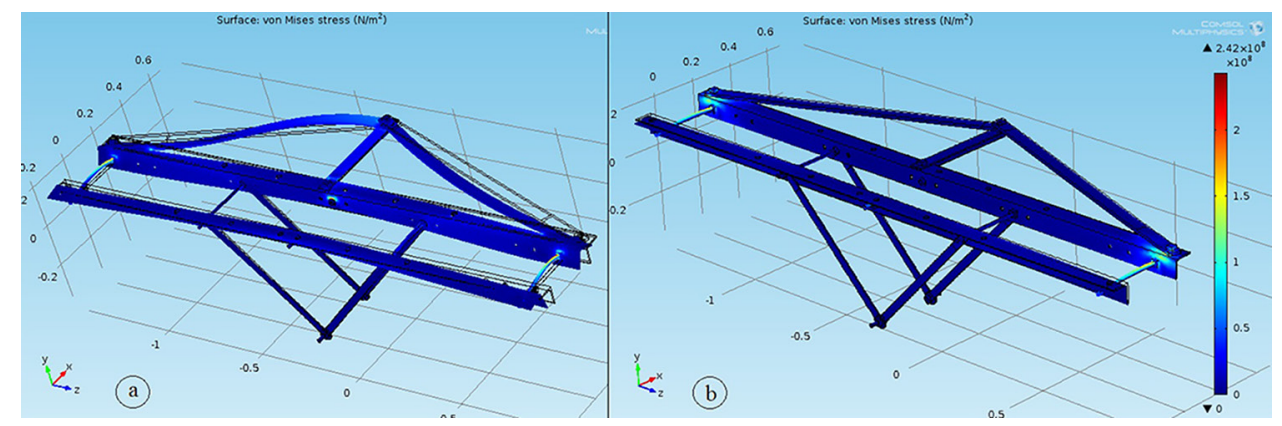

Figura 10. Modelado de condiciones críticas - remate pesado.

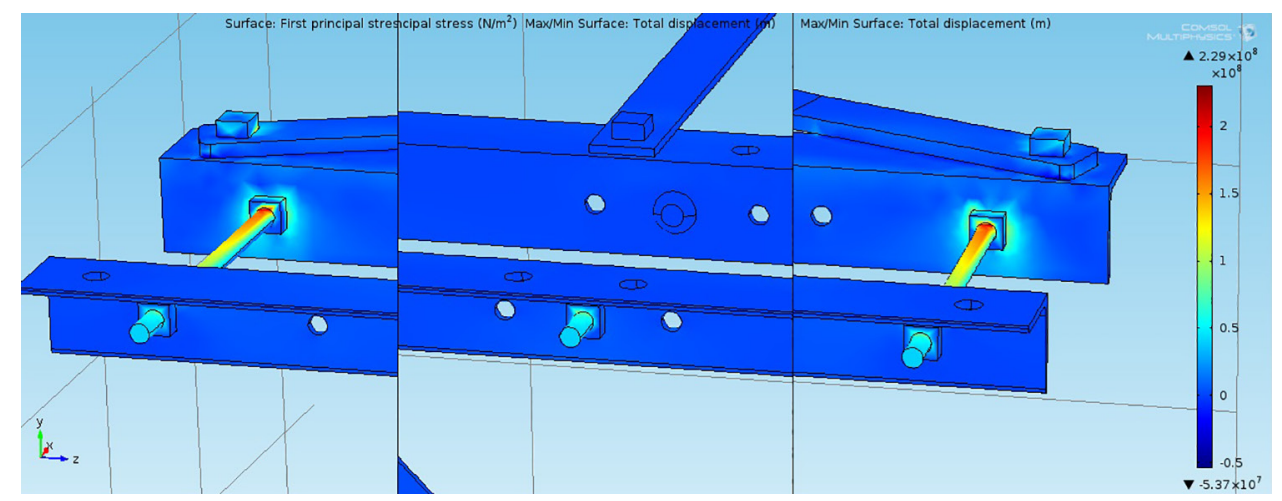

Figura 11. Principales resultados - remate pesado.

Con respecto a los herrajes, el mayor esfuerzo se presenta en el crucero, en las zonas cercanas a los pernos de armado doble de los extremos y corresponde a $93 \mathrm{MPa}$. En este montaje, bajo condiciones de sobrecarga, el mayor desplazamiento es de 3,14 mm y se presenta en uno de los extremos del crucero opuesto al soporte transversal.

En las tablas 3 y 4 se resumen los resultados obtenidos en el modelado de esfuerzo de todos los montajes trifásicos utilizados en la actualidad. Ahí se señala la zona donde se presentó el mayor esfuerzo de tensión y la magnitud del mismo así como el desplazamiento máximo.

Como se puede detallar en las tablas 3 y 4 , todos los herrajes operan actualmente bajo los márgenes de seguridad recomendados por el NESC (2002) y adoptados como criterio para este trabajo (250 MPa). En el caso de los montajes livianos los esfuerzos son en promedio inferiores al $32 \%$ de la capacidad de los herrajes, demostrándose que existen importantes oportunidades de mejora en el aprovechamiento de los montajes, respaldando la propuesta de nuevos diseños. En el caso de los montajes pesados, el esfuerzo máximo en los herrajes es en promedio inferior al $60 \%$ de la capacidad de esos 
elementos (250 MPa), por lo que también se presentan oportunidades de mejora que se incluirán en los diseños propuestos. En conjunto, los 12 montajes actuales presentan en condiciones de sobrecarga un esfuerzo máximo promedio de $114 \mathrm{MPa}$ en los herrajes, representando esto un uso de $45 \%$ la capacidad de dichos elementos.

Tabla 3. Resumen de resultados, montajes livianos.

\begin{tabular}{|c|c|c|c|}
\hline Montaje & Zona de mayor esfuerzo & $\begin{array}{l}\text { Esf. Máx. } \\
(\mathrm{MPa})\end{array}$ & $\begin{array}{l}\text { Desp. Máx. } \\
\quad(\mathrm{mm})\end{array}$ \\
\hline Paso de $0^{\circ}$ a $10^{\circ}$ & $\begin{array}{l}\text { Parte superior del crucero entre los aisladores más lejanos } \\
\text { al poste }\end{array}$ & 60 & 8,04 \\
\hline \multirow{2}{*}{ Paso de $10^{\circ}$ a $30^{\circ}$} & Parte superior del crucero en las cercanías al aislador central & 55 & \multirow{2}{*}{4,78} \\
\hline & Perno de armado doble central & 81 & \\
\hline $\begin{array}{l}\text { Paso de } 0^{\circ} \text { a } 10^{\circ} \\
\text { en bandera }\end{array}$ & Soporte para levantar el aislador & 54 & 4,59 \\
\hline \multirow{2}{*}{$\begin{array}{l}\text { Paso de } 10^{\circ} \text { a } 30^{\circ} \\
\text { en bandera }\end{array}$} & Sección de los arriostres cercana a la unión con los cruceros & 64 & \multirow{2}{*}{3,65} \\
\hline & Pernos de armado doble centrales & 105 & \\
\hline \multirow{2}{*}{ Abertura } & Crucero en las cercanías a pernos extremos & 86 & \multirow{2}{*}{1,16} \\
\hline & Pernos centrales & 186 & \\
\hline \multirow{2}{*}{ Remate } & Zona central de uno de los cruceros & 158 & \multirow{2}{*}{4,04} \\
\hline & Pernos de armado doble extremos & 312 & \\
\hline
\end{tabular}

Tabla 4. Resumen de resultados, montajes pesados.

\begin{tabular}{|c|c|c|c|}
\hline Montaje & Zona de mayor esfuerzo & $\begin{array}{l}\text { Esf. Máx. } \\
\text { (MPa) }\end{array}$ & $\begin{array}{l}\text { Desp. Máx } \\
(\mathrm{mm})\end{array}$ \\
\hline Paso de $0^{\circ}$ a $10^{\circ}$ & $\begin{array}{l}\text { Zona superior del crucero cercana a la sujeción del } \\
\text { arriostre }\end{array}$ & 94 & 7,17 \\
\hline \multirow{2}{*}{ Paso de $10^{\circ}$ a $30^{\circ}$} & Zona inferior del crucero en el extremo cercano al poste & 125 & \multirow{2}{*}{9,01} \\
\hline & Perno de armado doble del extremo cercano al poste & 201 & \\
\hline $\begin{array}{l}\text { Paso de } 0^{\circ} \text { a } 10^{\circ} \\
\text { en bandera }\end{array}$ & Zona del crucero cercana a la sujeción con el arriostre & 198 & 32,9 \\
\hline \multirow{2}{*}{$\begin{array}{l}\text { Paso } 10^{\circ} \text { a } 30^{\circ} \\
\text { en bandera }\end{array}$} & $\begin{array}{l}\text { la parte superior del crucero, en las cercanías con los } \\
\text { arriostres }\end{array}$ & 161 & \multirow[t]{2}{*}{21,7} \\
\hline & Perno de armado doble más alejado del poste & 174 & \\
\hline \multirow{2}{*}{ Abertura } & Crucero en las cercanías a pernos extremos & 220 & \multirow{2}{*}{3,11} \\
\hline & Perno central donde se aplica la tensión & 350 & \\
\hline \multirow{2}{*}{ Remate } & Zona de crucero cercana a pernos de armado doble & 93 & \multirow{2}{*}{3,14} \\
\hline & Pernos que llevan la carga & 229 & \\
\hline
\end{tabular}


Para los pernos de armado doble, estos márgenes corresponden a $250 \mathrm{MPa}$ en condiciones de tensión y $320 \mathrm{MPa}$ en condiciones de flexión. De acuerdo a esto, se presenta un caso que sobrepasa los márgenes de seguridad mencionados, es el perno central del montaje en abertura tipo pesado, donde se presenta un esfuerzo de flexión de $350 \mathrm{MPa}$. A pesar de que el valor es superior, se debe tomar en cuenta que para el modelado se utiliza como punto de referencia (punto fijo) la cara interna de la cabeza del perno y en la realidad, esta sección tiene alguna flexibilidad que es la brindada por la abrazadera que sujeta el montaje por lo que no se espera que en la práctica se alcancen estos valores.

El máximo desplazamiento que se presenta en los montajes es inferior a 3,3 cm valor que no afecta en ninguna medida el funcionamiento de los montajes.

\subsection{Esfuerzos en los pernos de sujeción de los montajes}

El análisis de equilibrio estático se realizó utilizando el software libre Force Effect de Autodesk. De acuerdo a lo establecido en el apartado 2.4, las fuerzas máximas que pueden soportar los pernos utilizados para la sujeción de los montajes son 36,45 kN de tensión y $14,65 \mathrm{kN}$ de cortante.

En la figura 12 se muestra el diagrama de cuerpo libre para el montaje paso de $0^{\circ}$ a $10^{\circ}$ liviano y el resultado obtenido para el caso de tensiones críticas de diseño (sobrecarga).

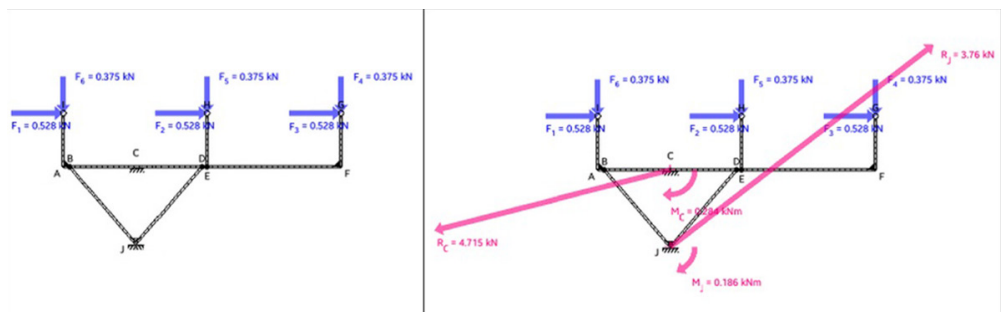

Figura 12. Equilibrio estático, paso $0^{\circ}$ a $10^{\circ}$ liviano.

Los resultados para los 12 montajes analizados se resumen en la tabla 5.

Como se aprecia, el montaje que transmite más tensión longitudinal a los pernos de soporte (y abrazaderas) es el remate pesado con 7,85 kN, valor considerablemente inferior a los $36,45 \mathrm{kN}$ establecidos como límite para los pernos. En condiciones de cortante, el que ejerce la mayor fuerza es la abertura pesada con $10,05 \mathrm{kN}$, valor inferior al límite establecido de $14,58 \mathrm{kN}$ para estos pernos.

\subsection{Modelado de la abrazadera}

En la figura 13-a se muestra la forma en que es instalada la abrazadera y sujetada al poste. Los pernos laterales de la brindan el ajuste entre las dos abrazaderas. Los pernos superior e inferior, son los utilizados para la sujeción de herrajes. En la figura 13-b se muestra el diagrama de cuerpo libre de una abrazadera. La fuerza T corresponde a la ejercida por el montaje a la abrazadera, las reacciones $\mathrm{R}$ son las que se dan en los pernos 
de sujeción y el esfuerzo distribuido D es la reacción del poste sobre la abrazadera cuando se aplica la tensión T.

Tabla 5. Reacciones en los soportes de los montajes actuales.

\begin{tabular}{|c|c|c|c|}
\hline Montaje & Perno & $\begin{array}{l}\text { Fuerza de tensión o } \\
\text { compresión }^{(-)}(\mathrm{kN})\end{array}$ & $\begin{array}{l}\text { Fuerza cortante } \\
(\mathrm{kN})\end{array}$ \\
\hline \multirow{2}{*}{ Paso $0^{\circ}$ a $10^{\circ}$ - liviano } & Superior & 0,00 & 3,76 \\
\hline & Inferior & 0,00 & 4,72 \\
\hline \multirow{2}{*}{ Paso $0^{\circ}$ a $10^{\circ}$ - pesado } & Superior & 0,00 & 6,88 \\
\hline & Inferior & 0,00 & 0,15 \\
\hline \multirow{2}{*}{ Paso $10^{\circ}$ a $30^{\circ}$ - liviano } & Superiores & 0,00 & 5,87 \\
\hline & Inferiores & 0,00 & 0,22 \\
\hline \multirow{2}{*}{ Paso $10^{\circ}$ a $30^{\circ}$ - pesado } & Superiores & 0,00 & 8,90 \\
\hline & Inferiores & 0,00 & 3,65 \\
\hline \multirow{2}{*}{ Paso $0^{\circ}$ a $10^{\circ}$ bandera - liviano } & Superior & 0,00 & 3,27 \\
\hline & Inferior & 0,00 & 2,05 \\
\hline \multirow{2}{*}{ Paso $0^{\circ}$ a $10^{\circ}$ bandera - pesado } & Superior & 0,00 & 8,49 \\
\hline & Inferior & 0,00 & 5,36 \\
\hline \multirow{2}{*}{ Paso $10^{\circ}$ a $30^{\circ}$ bandera - liviano } & Superiores & 0,00 & 3,99 \\
\hline & Inferiores & 0,00 & 1,96 \\
\hline \multirow{2}{*}{ Paso $10^{\circ}$ a $30^{\circ}$ bandera - pesado } & Superiores & 0,00 & 10,05 \\
\hline & Inferiores & 0,00 & 4,66 \\
\hline \multirow[t]{2}{*}{ Abertura - liviano } & Superiores & 0,00 & 4,62 \\
\hline & Inferiores & 0,00 & 1,16 \\
\hline \multirow{2}{*}{ Abertura - pesado } & Superiores & 0,00 & 8,01 \\
\hline & Inferiores & 0,00 & 2,29 \\
\hline \multirow{3}{*}{ Remate - liviano } & Frontal $^{1}$ & 6,05 & 0,19 \\
\hline & Posterior & $-3,03$ & 0,19 \\
\hline & Inferiores & 0,00 & 0,40 \\
\hline \multirow{3}{*}{ Remate - pesado } & Frontal $^{1}$ & 7,85 & 0,80 \\
\hline & Posterior & $-15,63$ & 0,80 \\
\hline & Inferiores & 0,00 & 1,39 \\
\hline
\end{tabular}

${ }^{1}$ Se refiere al lado que soporta los conductores.

El modelado de la abrazadera y su unión al poste por el método de diferencias finitas presenta el inconveniente del grado de libertad hacia el giro del poste y principalmente, la variación de la superficie de contacto conforme aumenta la fuerza aplicada por el perno a la abrazadera (por la deformación que se presenta). Este fenómeno se puede apreciar en la figura 14-a, donde se muestra el resultado con factor de escala de 100. A pesar de esta limitante, se determinó que para la máxima tensión transferida por los montajes 
actuales a la abrazadera $(7,85 \mathrm{kN})$, la deformación que se presenta en las abrazaderas es prácticamente nula (menor a $0,2 \mathrm{~mm}$ ), por lo que el modelado de la abrazadera sin la reacción del poste (D) es válida para esfuerzos de tensión. En la figura14-b se muestra el resultado del modelado sin factor de escala.
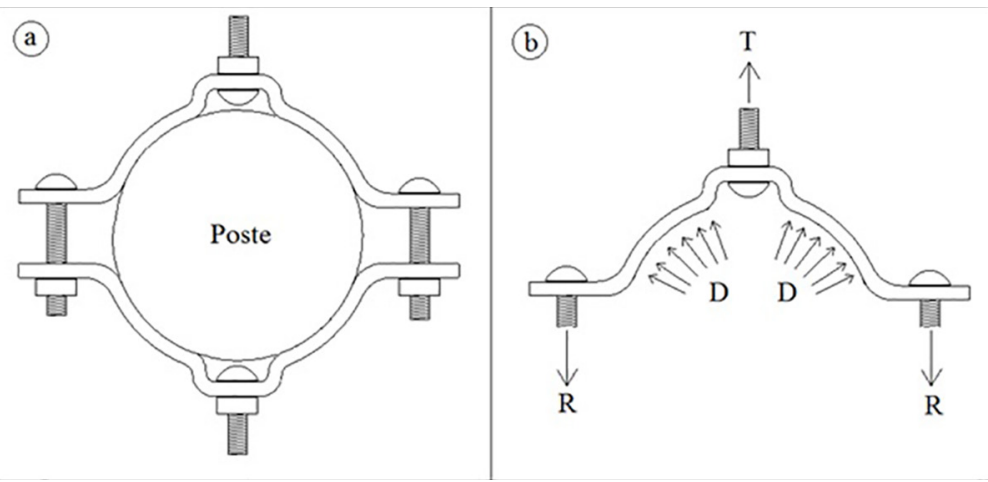

Figura 13. Diagrama de cuerpo libre de la abrazadera.

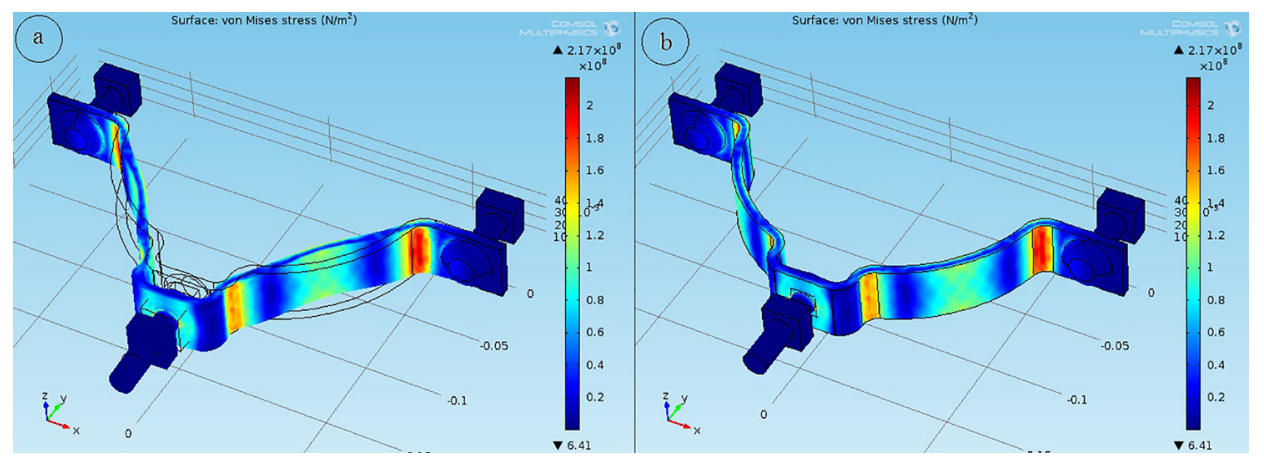

Figura 14. Modelado de la abrazadera con fuerza de tensión.

El máximo esfuerzo que ocurre en la abrazadera es de $217 \mathrm{MPa}$ y se da en la curvatura más cercana al perno de unión con la otra abrazadera.

Como se puede ver en la tabla 5, la mayor fuerza cortante sobre los pernos (y abrazaderas) tiene una magnitud apreciable comparada con la mayor tensión (25\% más). Para el modelado de esfuerzos de la abrazadera, cuando se transfiere por medio del perno una fuerza cortante de $10,05 \mathrm{kN}$ se consideró la reacción del poste denominando las caras internas de las abrazaderas como fijas (caso crítico). En la figura 15 se muestra el resultado del modelado de esfuerzos de ese escenario.

Como se puede ver, el máximo esfuerzo que se presenta en la abrazadera en este escenario es de 243 MPa y se da en la curvatura interna de la sección más cercana al perno. Este valor corresponde al $97 \%$ de la capacidad del acero por lo que, en caso de que se desee aumentar las cargas de los montajes tipo bandera pesados, deberá prestarse especial atención a la abrazadera. 


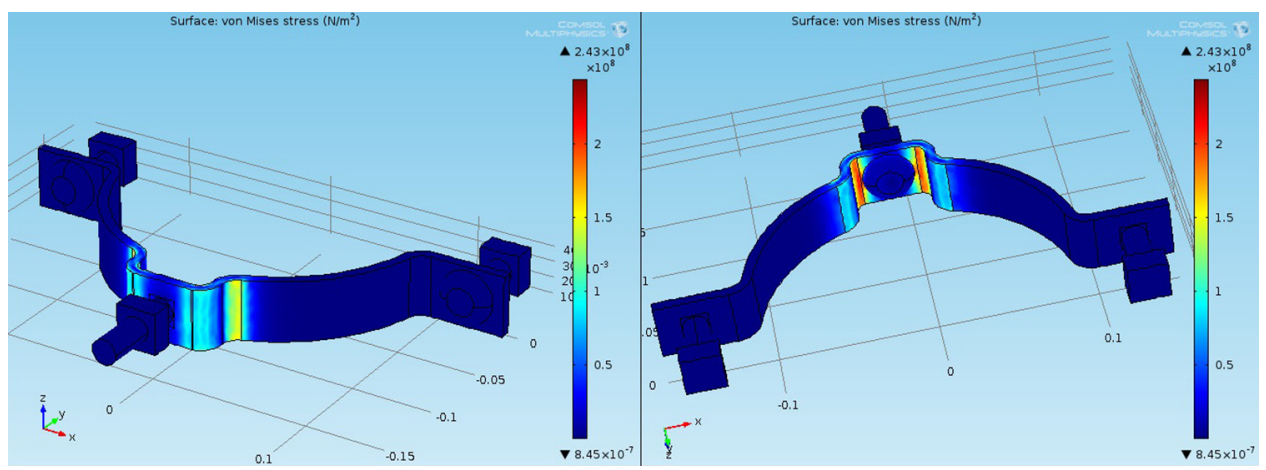

Figura 15. Modelado de la abrazadera con fuerza cortante.

\section{CONCLUSIONES}

De los materiales utilizados en los montajes de líneas de distribución, el uso de acero en la CNFL se considera el más adecuado (en comparación con el concreto o la madera) por su versatilidad de formación, ductilidad, tenacidad y condición de libre mantenimiento. No se encontró suficiente evidencia que justifique las distancias entre fases utilizadas en la CNFL para cada montaje, ni estudios relacionados al desempeño mecánico de los montajes que se utilizan en la actualidad.

Según el ángulo de desvío y aplicación específica, en la actualidad se utilizan doce tipos de montajes, con un cambio importante desde el punto de vista de la robustez del montaje cuando el ángulo supera los $10^{\circ}$ y $30^{\circ}$. No se encontró justificación técnica que determine el cambio de montaje a partir de esos ángulos, permitiendo eliminar ese cambio, estableciéndolo donde se justifique, en los nuevos diseños.

Acerca de los factores de seguridad utilizados para el diseño mecánico de líneas de distribución, la mayoría de fuentes consultadas utilizan un factor de seguridad referido a esfuerzos de rotura de los elementos sin mayor detalle al respecto. El Código de Seguridad de Estados Unidos, NESC, establece un factor de sobrecarga y otro de esfuerzo, brindando además detalles sobre los tipos de cargas y materiales, razón por la cual se tomó este estándar como referencia para la adopción de los factores de seguridad para el diseño mecánico de los montajes.

Para los montajes actuales, se determinó el estado de esfuerzos en condiciones máximas y de acuerdo a los criterios de diseño establecidos, obteniendo que el desempeño de todos es adecuado.

En el caso de los montajes livianos, en los herrajes se alcanza un esfuerzo de 158 MPa, representando $63 \%$ de su capacidad. Se puede afirmar que el diseño actual de estos montajes permite que sean utilizados para ángulos de desvío considerablemente mayores sin alcanzar su máxima capacidad. El desplazamiento producido por los esfuerzos máximos en los montajes, no es un factor relevante ni que merezca consideración en los diseños eléctricos, el máximo valor encontrado corresponde a $8 \mathrm{~mm}$. En el caso de 
los pernos, la condición de tensión crítica corresponde al montaje de remate y alcanza 90\% de su capacidad. Para los demás montajes, ese porcentaje es inferior a $53 \%$.

Para los montajes pesados, en los casos de paso (bandera y convencional) el mayor esfuerzo en los herrajes es de $80 \%$ por lo que para todos los casos podría ampliarse el ángulo de aplicación. De la misma manera, el máximo esfuerzo que se presenta en los pernos de esos montajes es de $57 \%$ por lo los pernos no limitarían el uso en ángulos mayores. En el caso del montaje de abertura, el esfuerzo máximo que se presenta en los herrajes es de $90 \%$ por lo que podría ampliarse en menor medida el ángulo de desvío, sin embargo, este aumento requeriría mayor análisis con respecto al acercamiento que pueda presentarse entre los conductores de distintas fases. También requeriría el uso de pernos con mayor capacidad mecánica en los extremos ya que para el caso del ángulo de desvío de $60^{\circ}$, los actuales alcanzan su máxima capacidad.

El análisis de equilibrio estático permitió determinar que los pernos de soporte de los montajes actuales son adecuados, alcanzando apenas $21 \%$ de su capacidad en tensión y $69 \%$ de su capacidad ante fuerzas cortantes. También permitió determinar que el montaje que más tensión transfiere a la abrazadera es el paso de $10^{\circ}$ a $30^{\circ}$ en bandera, el cual somete a la abrazadera a un esfuerzo de $97 \%$ de su capacidad. En caso de que este montaje sea utilizado para ángulos mayores, se deberá aumentar la capacidad de la abrazadera (aumentando su área de sección por ejemplo).

Se analizó la capacidad mecánica de los elementos que componen los montajes actuales y se determinó que los aisladores tipo poste vertical son mecánicamente subutilizados. En el caso de los montajes de uso liviano, para las condiciones de la CNFL estos aisladores podrían ser utilizados en un ángulo de desvío de hasta $129^{\circ}$. Para el caso de los montajes de uso pesado, un aislador de esta clase podría ser utilizado en ángulos de hasta $40^{\circ}$. En ambos casos, actualmente se utilizan hasta ángulos de desvío de $10^{\circ}$.

Existe una potencial oportunidad de mejora de los montajes que se utilizan en la actualidad. Mediante nuevos diseños o modificando en alguna medida los existentes, podría disminuirse el volumen efectivo de acero y reducir los costos.

\section{REFERENCIAS}

Dirección General de Electricidad, Ministerio de Energía y Minas (2003). Bases para el diseño de líneas y redes secundarias para electrificación rural. Perú.

Empresa de Energía de Boyacá S.A. (2010). Norma de diseño de redes de distribución de energía eléctrica. Colombia.

Empresa Provincial de Energía de Córdoba (1998). ET1002 Líneas áreas de Media Tensión $13,2 / 33 \mathrm{kV}$. Argentina.

Fernández, R. (2014). Criterios para el diseño mecánico de líneas de distribución de la CNFL. Costa Rica: Compañía Nacional de Fuerza y Luz, S.A.

Grupo ENERSIS (2002). Convergencia de Criterios de Diseño de Líneas Aéreas de Alta Tensión. Chile.

Ministerio de Industria, Turismo y Comercio (1968). Reglamento de Líneas Eléctricas Aéreas de Alta Tensión de España. España. 
NESC (2002). National Electrical Safety Code. USA: IEEE.

Shigley, J. E. y Mischke, C. R. (2002). Diseño en ingeniería mecánica. (Sexta Edición). USA: McGraw-Hill.

Soltis, L. A. and Wilkinso, T. L. (1996). Mechanical Connections in Wood Structures. USA: USDA Forest Product Laboratory. 